\title{
The Folk Flavor of Food of Dongting Lake Area in China
}

\author{
Fei-Long LIU ${ }^{1, a,{ }^{*}}$, Si-Yan DIN ${ }^{1, b}$ \\ ${ }^{1}$ School of Economics and Management Hunan Instituteof Science and Technology, \\ 414006, Yueyang, Hunan, China \\ aLiufeilong12@126.com, b601518668@qq.com
}

${ }^{*}$ Corresponding author

\section{Keyword: China, Dongting lake area, Folk flavor of food}

\begin{abstract}
DongTing Lake area is located in the north of Hunan province of china and known as the hometown of fish and rice. For a long time, "HuGuang cooked, the world is adequate," saying more widelycirculated. So it is certainly rich in products that can certainly promote the development of folk of rich and colorful food culture .Especially the DongTing Lake folk dishes Is the sound of people seat treasures with the various and beautiful. The characteristic of which are Rice noodle dishes series, Powder steamed dishes series , Jars kimchi series ,Game dishes series and Fish dishes series etc

"DongTing water all over the world, YueYang floor in all the world ". Wide of eight hundred in the DongTing lake is famous as the song verse. But DongTing lake diet folk a miracle of the flower in bud are like deep family, less known.Therefore, this study and explore nature has profound practical significance.DongTing lake area is located in the north of Hunan province, The Hunan belongs to the subtropical regions, Mild climate, four distinct seasons,Fertile land, abundant rainfall.ESW its territory surrounded by mountains and hills on three sides, Central Hill Gang and downs, several Pinnacle.The north was "800 Li Dongting Lake",Dongting Lake area is at the bottom of a giant horseshoe. It contain the thousands of people from the three area of YueYang city,YiYang city and ChangDe city. Because of a common geographical environment and climatic conditions, Thus despite minor differences here eating folk, but roughly the same.. DongTing lake, known as the hometown of fish and rice ,can also be known as "HuGuang cooked, the world is adequate".So it is certainly rich in products that can certainly promote the development of folk of rich and colorful food culture .Especially the DongTing lake folk dishes Is the sound of people seat treasures with the various and beautiful.The characteristic of which are Rice noodle dishes series ,Powder steamed dishes series, Jars kimchi series ,Game dishes series and Fish dishes series etc .[1][2][3]
\end{abstract}

\section{One of the Dongting Lake region diet flavor: rice noodle dishes series}

The rice food products taste flavor and chic, mouth at large .The representative dishes are Rice dumplings,Dragon Boat Festival,rice dumplings and sheet jelly packages of meat ,Hui braised pork and vermicelli, fresh rice noodle ect. Most of its production methods are quite exquisite Take Rice dumplings for example:first use clean water soak rice half a day ,then drain water use small fire fry till half cooked, leave to cool before hand whet into thick rice noodles, add the right amount of water at this time, tune into a paste, pinched by gathering and not glue the hand .Finally,take the meat or other kinds of Caixian having been done before into the steamed stuffed bun inside like the size of dumpling, then put it into steamer and steam for thirty minutes to eat.There are some characteristics:savoury,mellow and oil but not greasy. Among neighborhoods, they can regard it as festival gifts. At the same time, it can increase a little festival atmosphere.Most importantly, using it to serve guest can reflect hospitality and more enthusiasm of peoples of DongTing lake.

\section{Two of the Dongting Lake region diet flavor: Powder steamed dishes series}

Most of the series of dishes are long-term spread among folk of DongTing Lake,but there are a few such as powder steamed vegetables, which are borrowed from HuNan Province and the northern border HuBei HongHu ,BaiLuo.The typical of these series are:steamed pork with rice 
flour,steamed porkchop,steamed taro,steamed eggplant,steamed garden chrysanthemum ,steamed celery and so on .Like the steamed pork with rice flour, which is often eaten by the people living near lakes.The way of cooking it is to cut the pork into meat about five inch long,then add amount of cooking wine, salt, soy ,soy sauce, pepper powder .Firstly ,pickled and then fry rice and red pepper in an iron into golden powder.Later,marinated meat mixing in the pepper rice,putting them into the steamer steam about medium heat for 45 minutes .Finally,boring for a few minutes can dish up the plate.The other way of steamed dishes are almost the same as the steamed pork with rice flour. Just steamed all kinds of vegetables into a more advance put fresh vegetable in wastewater waterlogging in the process of the water.

\section{Three of the Dongting Lake region diet flavor: Jar of pickles Series}

People in the DongTing Lake area with their exquisite workmanship ,through pottery jars,ceramic cylinder,make all kinds of picked vegetables become delicious and different and their own style.Such as :Chili carrot, chopped pepper.sweet and sour chin ,sweet and sour beams, sweet and sour sword beam,salty cucumber, salt jerusalem artichoke .Meanwhile,there are also sorts of dry salt dish, semi-rotted vegetables etc.Their productive method is not difficult, such as the productive of chili carrot:one of the methods is to fresh white carrot and then cut them into strips,sun dried turnip into half,add chopped pepper and salt prepared mixing together string well into the alter cylinder inside, last seal them for about twenty days ,then can take it out to eat.The productive of dried carrot not always has tenality, but also chew sweaty including sweet,salty,spicy,aftertaste endless. The second way :cut the carrots into strips,stir the carrots ,pepper,salt together,and them put them in a sealed jar.After twenty days ,it can be tasted.[4]

\section{Four of the Dongting Lake region diet flavor: Lakes game series}

Characteristics of DongTing Lake area game and some have been mining on the end table,while others cannot be mined to protect the natural development .There series main dishes include duck stew of carrots ,dyke artemisics fried bacon ,pork chop stew lake lotus root,fried chili with black beams and wild celery , asparagus stir fry pork with artemisia tip slip and shredded pork and so on .Especially upscale flavor on the embankment of artemisia fried bacon wrapped side table when entertaining guests ,all were highly praised.With the improvement of this popular game is worth ,lake Artemisia felt dike in DongTing Lake area will become the leader on the market, only the south sales every year thousands of tons of Artemisia Dyke in YueYang city .Home of Artemisia visitors may bring along Dyke in YueYang. Dike Artemisia fried bacon can be cooked as follows:artemisia fresh levee washes cut into about one inch and then ironed in the boiling water, then cut the bacon into thin slices, put the bacon in the pot after the oil is exceeded,we should embankment of Artemisia ,put together a little pepper, monosodium glutamate, cooked four to eight minutes.

\section{Five of the Dongting Lake region diet flavor: Dongting Lake fish flavor Series}

This is the most unique dish series in DongTing lake area .People can cook hundreds of fish dishes with the smell of the fish of DongTing lake,such as halal fish, sweet fish and sour fish,bamboo fish ,fish balls, dry fried fish,sliver fish .fried eel slices ,dry cooked fish genus ,vanmanenia from GuangXi ,turtle with brown sauce ,steamed eel with and so on .We listed the smell of fish dishes in DongTing Lake area, it is just a small part.According to the survey,there are others:run snow spring,stars moons, butterflies crossing the harbour ,halal fish, fried needle beak.

Whitebait and red dates soup, Hot and sour small shrimp soup, Fish tofu soup, Baked fish, Hot and sour soup phoenix-tailed, and so on, there are also various jar products, such as Powder residue shrimp, Lees fish, they are the unique flavor. Some of the cooking methods of these folk fish are easy, and some of them are difficult, such as the dish of Ruixue spring, take some fresh catfish or back fish, remove their linings, put the diced into the container, add appropriate amount of salt, dry 
out after about 4 hours, then soft deep-fried in lard, remove and put them in the tray after frying into a milky white, and put the chopped green onion, ginger, chili powder over it, and then you can eat it. The characteristic of this dish is: crisp refreshing, fish infinite.[5]

Some flavor fish dishes get a bit of legend in cooking, such as "Loach drilling tofu fish", It is cooked in this way: Put the loach which has been spit water ,that is, put the loach which has been fed in the water in a few days, into pot, and at the same time put tender tofu into the block, now slowly heat up with a small fire, small loach realize that water temperature in the pot is rising gradually, then scramble desperately into tender tofu, when the temperature of the pot increases to a certain degree, small loach have already all scrambled into the tofu, at this time put in all kinds of seasoning and salt, after the soup boiling about 10 minutes, then you can eat it. This dish is not only delicious but also nutritious, when people put pieces of tofu which scrambled with small loach into the mouth, they praise its interesting cooking methods.

The lake district has a lot of delicious food, it also associate the food culture with the tourism culture subtly, so it has a strong aesthetic appeal. Such as the flavor fish dish "Dragon lady and A Bin Of Pearls", which is so beautiful from the shape. A red carp, fish body and the stomach full fill with Xiang Lian meat, whose shape look like a pearl, ornamented with parsley, red and white, flanked by green shade, its taste and nutrition are good. But it is not only the most attractive, it also has a very beautiful legend. It is said that long time ago, xiu cai liuyi of Dong ting Lake District went to the capital for the imperial examination, he encountered the dragon king's three daughter, who suffered a lot in the world, so he risked sending letters to help Three Princess, in order to show thanks for the favour of liuyi's help, Three Princess ordered the chef to do expensive banquet, one of the fish dishes, its fish body and the stomach full fill with first-rate pearl. On the other hand, Dragon lady want to use it to thank her benefactor, on the other hand, she want to show her love, using the dish "Dragon lady and A Bin Of Pearls" (partial tone) to extend her full love to childe liu. So people evolved this delicious dish, which filled with touching love story, into "Dragon lady with A Stomach Of Pearls".[6]

Since the end of last century, exploiting folk flavor food of Dong ting Lake District has aroused attention of the relevant persons. For example, the native yue yang chef create "Dong Ting whole fish seats"(Also known as "ba ling whole fish seats") on the basis of collecting large amounts of folk flavor food, which is well-known both in China and in foreign country. This dish has been listed in the "Chinese Recipes", but the degree of exploitation is not enough on the whole. Dongting Lake area folksy diet in terms of its economic value, nutritional value and ornamental value, or its cultural connotation has its own unique, worthy of our careful excavation and development.

\section{References}

[1] Zhongyi Zhang, Dongting Lake area in ancient times on Regional Culture, J.Journal of Yueyang Vocational and Technical College. 1(2010).

[2] Ronghua Nie, Li Wan , Huxiang culture introduction, M.Hunan University press. 2005.

[3] Xueping Wang ,The regional characteristics of the pre Qin food culture, J.Qinghai Social Sciences. 4 (2006).

[4] Guanzhong Shen,Talking seasoning , J.Cuisine Journal of Yangzhou University.2 (1995 ).

[5] Hongkun Ji. Diet beauty and diet flavor, J.Cuisine Journal of Yangzhou University.1(2005).

[6] Feilong Liu, Baling whole fish Historical Status and Future Development, J. Journal of Yunmeng,.2 (2011). 